University of Wollongong

Research Online

Faculty of Engineering and Information

Faculty of Engineering and Information

Sciences - Papers: Part A

Sciences

$1-1-2013$

Comparison between sequential and simultaneous application of activated carbon with membrane bioreactor for trace organic contaminant removal

Luong N. Nguyen

University of Wollongong, Inn909@uowmail.edu.au

Faisal I. Hai

University of Wollongong, faisal@uow.edu.au

Jinguo Kang

University of Wollongong, jkang@uow.edu.au

Long D. Nghiem

University of Wollongong, longn@uow.edu.au

William E. Price

University of Wollongong, wprice@uow.edu.au

See next page for additional authors

Follow this and additional works at: https://ro.uow.edu.au/eispapers

Part of the Engineering Commons, and the Science and Technology Studies Commons

Research Online is the open access institutional repository for the University of Wollongong. For further information contact the UOW Library: research-pubs@uow.edu.au 


\title{
Comparison between sequential and simultaneous application of activated carbon with membrane bioreactor for trace organic contaminant removal
}

\begin{abstract}
The removal efficiency of 22 selected trace organic contaminants by sequential application of granular activated carbon (GAC) and simultaneous application of powdered activated carbon (PAC) with membrane bioreactor (MBR) was compared in this study. Both sequential application of GAC following MBR treatment (MBR-GAC) and simultaneous application of PAC within MBR (PAC-MBR) achieved improved removal (over $95 \%$ ) of seven hydrophilic and biologically persistent compounds, which were less efficiently removed by MBR-only treatment (negligible to 70\%). However, gradual breakthrough of these compounds occurred over an extended operation period. Charged compounds, particularly, fenoprop and diclofenac, demonstrated the fastest breakthrough (complete and 50-70\%, in MBR-GAC and PAC-MBR, respectively). Based on a simple comparison from the long-term performance stability and activated carbon usage points of view, PAC-MBR appears to be a better option than MBR-GAC treatment.
\end{abstract}

\section{Keywords}

organic, contaminant, removal, carbon, membrane, activated, bioreactor, application, simultaneous, sequential, between, comparison, trace

\section{Disciplines \\ Engineering | Science and Technology Studies}

\section{Publication Details}

Nguyen, L. N., Hai, F. I., Kang, J., Nghiem, L. D., Price, W. E., Guo, W., Ngo, H. H. \& Tung, K. (2013). Comparison between sequential and simultaneous application of activated carbon with membrane bioreactor for trace organic contaminant removal. Bioresource Technology, 130 412-417.

\section{Authors}

Luong N. Nguyen, Faisal I. Hai, Jinguo Kang, Long D. Nghiem, William E. Price, Wenshan Guo, Huu H. Ngo, and Kuo-Lun Tung 


\title{
Comparison between sequential and simultaneous application of activated carbon with membrane bioreactor for trace organic contaminant removal
}

\author{
Bioresource Technology 130 (2013) 412-417 \\ Luong N. Nguyen ${ }^{a}$, Faisal I. Hai ${ }^{a}{ }^{*}$, Jinguo Kang ${ }^{a, b}$, Long D. Nghiem ${ }^{a}$,William E. Price ${ }^{b}$, \\ Wenshan Guo ${ }^{c}$, Hao H. Ngo ${ }^{c}$ and Kuo-Lun Tung d,e \\ ${ }^{\text {a }}$ Strategic Water Infrastructure Laboratory, School of Civil, Mining and Environmental \\ Engineering, University of Wollongong, Wollongong, NSW 2522, Australia \\ ${ }^{\mathrm{b}}$ Strategic Water Infrastructure Laboratory, School of Chemistry, University of Wollongong, \\ Wollongong, NSW 2522, Australia \\ ${ }^{\mathrm{c}}$ Centre for Technology in Water and Wastewater, School of Civil and Environmental \\ Engineering, University of Technology Sydney, Sydney, Broadway, NSW 2007, Australia \\ ${ }^{\mathrm{d}}$ Department of Chemical Engineering, National Taiwan University, Roosevelt Rd, Taipei 106, \\ Taiwan \\ ${ }^{\mathrm{e}}$ R\&D Center of Membrane Technology, Chung Yuan University, Chung-Li 320, Taiwan
}

* Corresponding author: Faisal I. Hai, Email: faisal@uow.edu.au, Ph +61 242213054 


\section{RESEARCH HIGHLIGHTS:}

- $\quad>95 \%$ removal of all trace organics was achieved by MBR-GAC and PAC-MBR initially

- Gradual drop in removal of some hydrophilic compounds occurred in both systems

- Known persistent compound carbamazepine showed high removal in both systems

- Charged compounds fenoprop and diclofenac showed high persistence in both systems

- $\quad$ PAC-MBR outperformed MBR-GAC in terms of adsorbent consumption 


\section{Abstract}

The removal efficiency of 22 selected trace organic contaminants by sequential application of granular activated carbon (GAC) and simultaneous application of powdered activated carbon (PAC) with membrane bioreactor (MBR) was compared in this study. Both sequential application of GAC following MBR treatment (MBR - GAC) and simultaneous application of PAC within MBR (PAC - MBR) achieved improved removal (over 95\%) of seven hydrophilic and biologically persistent compounds, which were less efficiently removed by MBR-only treatment (negligible to 70\%). However, gradual breakthrough of these compounds occurred over an extended operation period. Charged compounds, particularly, fenoprop and diclofenac, demonstrated the fastest breakthrough (complete and 50-70\%, in MBR - GAC and PAC - MBR, respectively). Based on a simple comparison from the long-term performance stability and activated carbon usage points of view, PAC - MBR appears to be a better option than MBR GAC treatment.

Keywords: Membrane bioreactor; powdered activated carbon; granular activated carbon; trace organic contaminants 


\section{Introduction}

In view of the potential adverse effects of trace organic contaminants on human health and that of other biota, numerous studies have been devoted for their removal from wastewater by membrane bioreactors (MBRs). The reported data have demonstrated better and/or more stable removal of trace organic contaminants of moderate to high biodegradability and/or significant hydrophobicity by MBRs as compared to conventional activated sludge (CAS) processes . However, significant variations in removal of hydrophilic and biologically persistent compounds by MBR have been noted in several recent studies (Bernhard et al., 2006; Visvanathan et al., 2005). Besides biological processes, adsorption onto powdered activated carbon (PAC) or granular activated carbon (GAC) can also be used to efficiently remove trace organic contaminants from water (Ternes et al., 2002). However, limited adsorption of ionic compounds, particularly of those containing electron-withdrawing functional groups, has been reported (Ternes et al., 2002). In this connection, the concept of combined processes such as coupling of MBR with PAC/GAC has been explored (Li et al., 2011; Lipp et al., 2012; Nguyen et al., 2012). Activated carbon adsorption in conjunction with an MBR can be applied in two different configurations: i) direct addition of PAC into MBR (PAC - MBR), and ii) post-treatment of MBR permeate by passing it through a GAC column (MBR - GAC) or by dosing of PAC. It is envisaged that due to the complete retention of sludge by membrane, the trace organic contaminants adsorbed onto PAC may be efficiently removed by a PAC - MBR (Li et al., 2011). On the other hand, because MBR can produce suspended solids-free permeate with low total organic carbon content, GAC can specifically target the residual trace organic contaminants in MBR permeate with reduced interference from the bulk organics (Nguyen et al., 2012). 
A few studies have explored PAC-MBR for the removal of trace organic contaminants (Li et al., 2011; Serrano et al., 2011; Zhang et al., 2008). Several studies have also explored adsorption of trace organic contaminants on either PAC (Lipp et al., 2012) or GAC (Nguyen et al., 2012) as a post treatment following MBR treatment. Previously reported data have confirmed instant improvement of removal of selected biologically persistent trace organic contaminants from liquid phase by combined MBR—activated carbon systems. However, a comprehensive understanding of the phenomena involved based on long-term operation is yet to be achieved. In particular, no study has specifically compared the trace organic contaminant removal performance of MBR - GAC and PAC - MBR.

This study systematically compared the removal efficiency of 22 selected trace organic contaminants by sequential application of GAC and simultaneous application of PAC with MBR (MBR - GAC and PAC - MBR, respectively). Compounds showing extraordinary resistance towards MBR treatment and their extent of removal by the combined systems were given special focus. The two options were compared on the basis of performance stability and activated carbon consumption.

\section{Materials and Methods}

\subsection{Trace organic contaminants}

A set of 22 trace organic contaminants were selected for investigation based on two criteria: i) their representation of four major groups of trace organic contaminants, namely, pharmaceutically active compounds, steroid hormones, pesticides and industrial chemicals and metabolites (Supplementary Data Table S1), and, ii) their widespread occurrence in natural water bodies polluted with wastewater (Kim et al., 2007; Ternes et al., 2002). The compounds were 
purchased from Sigma-Aldrich (St. Louis, MO, Australia). The purity of these chemicals was reported to be $98 \%$ or higher. The selected trace organic contaminants were first dissolved in pure methanol to make up stock solutions of $1 \mathrm{~g} / \mathrm{L}$. The stock solutions were stored at $-18^{\circ} \mathrm{C}$ and were used within 1 month. The trace organic contaminants were introduced to the feed solution to achieve a constant concentration of approximately $5 \mu \mathrm{g} / \mathrm{L}$ of each compound.

\subsection{Synthetic wastewater}

A synthetic wastewater containing glucose $(400 \mathrm{mg} / \mathrm{L})$, peptone $(100 \mathrm{mg} / \mathrm{L}), \mathrm{KH}_{2} \mathrm{PO}_{4}(17.5$ $\mathrm{mg} / \mathrm{L}), \mathrm{MgSO}_{4}(17.5 \mathrm{mg} / \mathrm{L}), \mathrm{FeSO}_{4}(10 \mathrm{mg} / \mathrm{L}), \mathrm{CH}_{3} \mathrm{COONa}(225 \mathrm{mg} / \mathrm{L})$ and $\mathrm{CO}\left(\mathrm{NH}_{2}\right)_{2}(35$ $\mathrm{mg} / \mathrm{L}$ ) was used. The synthetic wastewater simulated medium strength wastewater with a total organic carbon (TOC), total nitrogen (TN) and chemical oxygen demand (COD) of approximately 180, 25 and $600 \mathrm{mg} / \mathrm{L}$, respectively. The synthetic wastewater was prepared freshly each day from concentrated stock solution and fed into the reactor.

\subsection{Activated carbon}

In this study, two types of activated carbon namely GAC 1200 and PAC 1000 (Activated Carbon, Technologies Pty Ltd, Victoria, Australia), with a specific surface area of 1121 and 1355

$\mathrm{m}^{2} / \mathrm{g}$, respectively were used. The characteristics of each type of activated carbon are listed in the Supplementary Data Table S2. Activated carbon was washed with Milli-Q water to remove fine particles, and then dried at $105^{\circ} \mathrm{C}$ for $24 \mathrm{~h}$ and stored until use. 


\subsection{MBR—activated carbon systems and experimental protocols}

\subsubsection{Design of MBR and GAC column}

A laboratory scale MBR set-up (Supplementary Data Figure S3) with an active volume of $4.5 \mathrm{~L}$ and equipped with a PVDF hollow fibre membrane module (Mitsubishi Rayon Engineering, Japan), was employed in this study. A borosilicate glass column (Omnifit, Danbury, CT, USA) filled with $7.5 \mathrm{~g}$ of GAC was used as a post treatment unit for the MBR permeate (Nguyen et al., 2012). The column had an internal diameter of $1 \mathrm{~cm}$ and an active length of $22 \mathrm{~cm}$, resulting in a bed volume (BV) of $17 \mathrm{~mL}$.

\subsubsection{Experimental protocol}

The current study was conducted over total 306 days, with 51 days of initial start-up period, 93 days of operation in MBR - GAC mode, 100 days of operation in PAC-MBR mode, and rest of the period in MBR-only operation mode. The exact sequence of different operations has been listed in Supplementary Data Table S4. The addition of the selected trace organic contaminants to the synthetic wastewater was started after the start-up period, when the mixed liquor suspended solids (MLSS) and mixed liquor volatile suspended solids (MLVSS) concentration were $5 \mathrm{~g} / \mathrm{L}$ and $4.5 \mathrm{~g} / \mathrm{L}$, respectively. The MBR system was operated for further 15 days before adding a GAC column as a post-treatment process for MBR permeate. During operation in MBR - GAC mode the MLSS concentration increased to $9.8 \mathrm{~g} / \mathrm{L}$. On day 158 , the GAC column was disconnected from the MBR and MBR operation was continued as usual. Sludge was withdrawn on day 197 to reduce the MLSS concentration to $6 \mathrm{~g} / \mathrm{L}$ prior to addition of PAC. PAC was added into the reactor on day 206 and subsequently on day 243 of continuous operation to obtain PAC concentrations of 0.1 and $0.5 \mathrm{~g}$ PAC/L, respectively (Supplementary Data Table S4). 
The MBR was seeded with activated sludge from another laboratory scale MBR system, which had been used for trace organic contaminant removal for about 3 years. Dissolved oxygen (DO) concentration and temperature of the mixed liquor was maintained at above $3 \mathrm{mg} / \mathrm{L}$. The MBR was placed into a water bath to maintain the mixed liquor temperature at $22 \pm 0.1{ }^{\circ} \mathrm{C}$. The $\mathrm{pH}$ of the mixed liquor remained stable within the range of 7.2 - 7.5. To ensure that membrane fouling did not interfere in the observation of trace organic contaminant removal, a low average membrane flux of $0.07 \mathrm{~m} / \mathrm{d}$ was applied in this study. The membrane was operated on a $14 \mathrm{~min}$ "suction" and $1 \mathrm{~min}$ "relaxation" cycle, resulting in a hydraulic retention time (HRT) of $24 \mathrm{~h}$. However, transmembrane pressure (TMP) was continuously monitored using a high-resolution $( \pm 0.1 \mathrm{kPa}$ ) pressure sensor (SPER scientific 840064, Extech equipment Pty. Ltd, Victoria, Australia), and ex-situ backwash ( $\mathrm{NaOCl}$ solution with $500 \mathrm{mg}$ active chlorine per $\mathrm{L}$ ) of the membrane every 90 days was observed to keep the TMP stable. In the MBR - GAC configuration, the MBR permeate was pumped through the GAC column in an up-flow mode at a flow rate of $2.4 \mathrm{~mL} / \mathrm{min}$ (equivalent to $8.5 \mathrm{BV}$ per hour), resulting in an empty bed contact time (EBCT) of $7 \mathrm{~min}$. The PAC - MBR system was operated in the same fashion as that during the MBR operation.

\subsection{Analysis of trace organic contaminants and other basic parameters}

The performance of the MBR, MBR - GAC and PAC - MBR systems was compared mainly in terms of trace organic contaminants and TOC/TN removal efficiency. Operating parameters such as MLSS and MLVSS concentration, turbidity, sludge volume index (SVI) and specific oxygen uptake rate (SOUR) were also monitored to confirm process stability. These basic parameters were measured according to the standard methods. 
The concentration of target organic compounds in MBR influent and permeate samples (duplicate) was measured once a week by a previously reported analytical technique (Nguyen et al., 2012) involving solid phase extraction, derivatisation and quantitative determination by a Shimadzu GC/MS (QP5000) system equipped with a Shimadzu AOC 20i autosampler. A Phenomenex Zebron ZB-5 (5\% diphenyl-95\% dimethylpolysiloxane) capillary column (30 m $\times$ $0.25 \mathrm{~mm} \mathrm{ID}, \mathrm{d}_{\mathrm{f}}=0.25 \mu \mathrm{m}$ ) was used. The quantitative detection limits of this analytical method were compound specific and in the range from 1 to $20 \mathrm{ng} / \mathrm{L}$. Removal efficiency was calculated as $R=100 \times\left(1-\frac{C_{E f f}}{C_{I n f}}\right)$, where $\mathrm{C}_{\mathrm{Inf}}$ and $\mathrm{C}_{\mathrm{Eff}}$ are influent and effluent (permeate) concentrations of the trace organic compounds, respectively.

\section{Results and discussion}

\subsection{Process stability and TOC/TN removal}

The process stability in terms of basic water quality parameters, namely, TOC/TN removal and permeate turbidity, and operating parameters such as SVI, SOUR, MLSS/MLVSS concentration and TMP were monitored to ensure that the trace organic contaminant removal data was collected at stable conditions. As the MBR was inoculated with sludge from another MBR treating trace organic contaminants for over 3 years, and a synthetic wastewater was used to maintain a consistent influent composition (Section 2), the performance of the system was stable. Turbidity of permeate in all three systems was continuously observed to be below $0.2 \mathrm{NTU}$. The average SVI and SOUR values were stable at approximately $150 \mathrm{~mL} / \mathrm{g}$ and $20 \mathrm{mg} \mathrm{O}_{2} / \mathrm{g}$ MLVSS. $\mathrm{h}$, respectively over the operation period irrespective of the combination tested. MLSS concentration increased gradually from 3.2 to $9.8 \mathrm{~g} / \mathrm{L}$ during operation without addition of PAC. 
However, no significant changes in MLSS concentration were observed after PAC addition. The latter is in line with the observation by Seker et al. (1995) who reported slower sludge production rate in case of biofilm developed on activated carbon surface as compared to suspended biomass. The variation in MLSS concentration during operation without PAC did not have any impact on TOC/TN removal. In fact, TOC was removed with high efficiency (> $98 \%$ ) throughout the operating period (Table 1). The observed high TOC removal irrespective of the MLSS concentration is in good agreement with a previous study (Hai et al., 2011). Without a denitrification zone within MBR, the MBR only showed a TN removal less than $50 \%$ but considerably stable (Table 1). Stable TMP values between the ex-situ chemical cleaning intervals (data not shown) also indicated that severe membrane fouling did not occur, which ensured stability of the hydraulic performance of the membrane.

\section{[TABLE 1]}

\subsection{Trace organic contaminants removal with and without activated carbon}

Previous studies have demonstrated that MBRs can efficiently remove the hydrophobic and relatively biodegradable compounds, while significant variation in MBR performance has been observed for biologically persistent and hydrophilic compounds (Berhnard et al., 2006;

Visvanathan et al., 2005). Similar to the previous studies, our results confirm the high degree of removal (over $90 \%$ and up to $99 \%$ ) of all of the steroid hormones and alkyl phenolic surfactants and industrial chemicals (Table 2). Notably all these compounds possess high hydrophobicity $(\log \mathrm{D}>3.2$ at $\mathrm{pH}=7$, Supplementary Data Table 1$)$ and contain strong electron donor $-\mathrm{OH}$ group, which makes them more amenable to oxidative degradation (Tadkaew et al., 2011). The investigated pesticides and pharmaceutically active compounds were all hydrophilic (Supplementary Data Table 1) and demonstrated varying levels of removal (e.g. carbamazepine 
(15-50\%), diclofenac (4-26\%) and naproxen (30-60\%)) (Table 2). Particularly, seven compounds, namely, metronidazole, fenoprop, naproxen, ketoprofen, diclofenac, carbamazepine and pentachlorophenol, were identified to be removed at efficiencies lower than $70 \%$. Low and/or variable removal of these seven hydrophilic compounds can be attributed to the existence of strong electron withdrawing group(s) in their structures (Tadkaew et al., 2011). Our results are consistent with the results obtained from previous studies (Bernhard et al., 2006; Reif et al., 2008) where unstable removal of hydrophilic and biologically persistent compounds, despite stable TOC/TN removals, have been reported.

\section{[TABLE 2]}

High removal (over $95 \%$ ) of all 22 selected trace organic contaminants was observed in both MBR - GAC and PAC - MBR systems (Table 2). Of particular interest was the significantly improved removal of the seven problematic compounds identified above. The initial high degree of removal efficiency achieved in MBR - GAC and PAC - MBR systems demonstrated that both GAC and PAC can enhance the trace organic contaminant removal performance of MBR. This observation is in line with that in previous studies which have reported that GAC can be a viable tool for the elimination of trace organic contaminants from surface water or biologically treated wastewater (Ternes et al., 2002). Similarly, PAC has been reported to be a good adsorbent for trace organic contaminants. For example, Serrano et al. (2011) reported significant removal of compounds such as carbamazepine, naproxen and diclofenac by adding PAC into MBR. 


\subsection{Trace organic contaminant removal: MBR-GAC versus PAC-MBR}

\subsubsection{Comparative effectiveness of the processes}

Despite high initial removal, gradual drop in adsorption of trace organic contaminants on GAC column requires replacement or regeneration of GAC (Hernández-Leal et al., 2011; Nguyen et al., 2012). Gradual reduction in removal efficiency of trace organic contaminants by PAC MBRs has also been documented (Li et al., 2011; Serrano et al., 2011). For example, decrease in removal efficiency of carbamazepine and diazepam was reported by Serrano et al. (2011) after around three months of operation of an MBR with $1 \mathrm{~g}$ PAC per litre. In the current study, the 15 trace organic contaminants (nine hydrophobic and six hydrophilic compounds), which were well removed by MBR treatment (Table 2), maintained high removal throughout both MBR - GAC and PAC - MBR operations. On the other hand, the removal efficiency of all seven biologically persistent and hydrophilic compounds (Section 3.2) by both MBR - GAC and PAC - MBR systems gradually dropped (Figure 1).

\section{[FIGURE 1]}

Since PAC and GAC were used in two different configurations in this study, and the use of a higher mass of GAC (compared to PAC) in the GAC column was inevitable, it is not possible to compare the performance of these two configurations based on the same mass of adsorbents. As a result, comparison has been made based on the treated volume (Figure 1). At a treated volume of $135 \mathrm{~L}, \mathrm{PAC}-\mathrm{MBR}(0.5 \mathrm{~g} / \mathrm{L}$ PAC) outperformed MBR - GAC. The better performance of PAC - MBR over MBR - GAC can be attributed to the higher surface area per unit weight of PAC (Supplementary Data Table S2). In addition, due to the larger particles size as well as the characteristic arrangement of GAC in a packed column, mass transfer hindrance may be severer 
in the GAC system. The trace organic contaminant removal performance of MBR - GAC and PAC - MBR systems has not been compared in the available literature. However, the performance of MBR - GAC and PAC - MBR in terms of oily wastewater treatment was compared in a study by William et al. (2009), who reported that PAC - MBR was better than MBR - GAC system in terms of effluent quality, less frequent membrane cleaning, tolerance to upsets and immediate acclimation.

Although at the PAC dose of $0.1 \mathrm{~g} / \mathrm{L}$, the PAC-MBR system showed the lowest removal

efficiency (Figure 1), for a valid comparison between two doses of the same adsorbent (PAC) in the same reactor, treated volume per unit weight of adsorbent needs to be considered. Further discussion on this point is available in Section 3.3.3.

\subsubsection{Compound-specific breakthrough profiles}

Generally, breakthrough profiles provide important information for subsequent regeneration of the spent activated carbon in GAC column or fixing dosage/withdrawal frequency of PAC in MBR. From cost considerations, for full scale installations, only selective marker compounds, not all, can be monitored. Thus, breakthrough profiles may help to identify the critical compounds which can be used as a marker. Because the higher dose of PAC ( $0.5 \mathrm{~g} / \mathrm{L})$ allowed observation of breakthrough behaviour for longer period, the comparison of breakthrough behavior of seven problematic compounds has been made between PAC - MBR ( $0.5 \mathrm{~g} / \mathrm{L})$ and MBR - GAC (Figure 2).

\section{[FIGURE 2]}

In this study, significant variations in the breakthrough profiles amongst seven hydrophilic and biologically persistent trace organic compounds were observed in MBR - GAC (Figure 2). The 
order of compounds in terms of decreasing severity of breakthrough was: fenoprop $\approx$ diclofenac

$>$ ketoprofen $>$ naproxen $>$ carbamazepine $>$ pentachlorophenol $>$ metronidazole, and notably, the breakthrough of the first four compounds exceeded 50\% within production of $120 \mathrm{~L}$ of effluent. On the other hand, in PAC - MBR $(0.5 \mathrm{~g} / \mathrm{L})$, the breakthrough of ketoprofen, carbamazepine, naproxen and pentachlorophenol remained stable within $20 \%$ over production of $280 \mathrm{~L}$ of effluent. The best removal of the neutral compounds carbamazepine and metronidazole by MBR - GAC may be because of their higher affinity towards GAC (Nguyen et al., 2012; Ternes et al., 2002). The higher removal of pentachlorophenol in both systems may be explained noting that this compound had been removed to a higher degree by MBR treatment as compared to the four other negatively charged problematic compounds (Table 2). Fenoprop and diclofenac showed particular resistance to both the systems due to their charge and significant hydrophilicity, indicating that they possess limited affinity towards activated carbon (Nguyen et al., 2012; Ternes et al., 2002). However, the reason of relatively lower removal of metronidazole and significantly higher removal of ketoprofen and naproxen, respectively in PAC - MBR could not be identified. Apart from the distinct properties of PAC and GAC (Supplementary Data Table S2), different loading of bulk organics and trace organic contaminants onto PAC and GAC may be responsible for the observed differences in removal. Nevertheless, the analysis of breakthrough profiles revealed that among the investigated compounds, fenoprop can be used as a marker for determination of period of regeneration/ replenishment of activated carbon.

\subsubsection{Performance comparison based on activated carbon usage}

Comparing the extent of breakthrough of fenoprop while taking into consideration the treated volume per unit weight of activated carbon $(\mathrm{L} / \mathrm{g})$, it is clear that the PAC - MBRs (both with 0.1 and $0.5 \mathrm{~g} / \mathrm{L}$ of PAC) outperformed MBR - GAC (Figure 3). Furthermore, it is interesting to note 
that under the tested conditions, up to an activated carbon usage rate of $120 \mathrm{~L} / \mathrm{g}$, similar levels of fenoprop removal could be achieved using PAC concentrations of 0.1 and $0.5 \mathrm{~g} / \mathrm{L}$. Immediately after adding into MBR, PAC can adsorb, in addition to trace organic contaminants, a wide range of bulk organic matter including products of microbial degradation and dead microbial cells $(\mathrm{Ng}$ et al., 2006). Our results suggest that the application of relatively frequent but smaller dosage of PAC is recommendable in order to minimize loss of adsorptive sites due to adsorption of bulk organics and consequently reduce overall PAC consumption..

\section{[FIGURE 3]}

In addition to the observation that a PAC - MBR system can outperform an MBR - GAC system in terms of adsorbent consumption (Figure 3), addition of PAC directly to MBR can also reduce membrane cleaning and/or membrane replacement frequency by retarding membrane fouling (Ng et al., 2006). Therefore, when all operating cost items i.e., activated carbon, membrane cleaning chemical, membrane replacement and energy consumption are taken into account, PAC - MBR is likely to appear more advantageous. However, a larger scale study is necessary for a detailed cost comparison.

\section{Conclusions}

PAC addition into MBR (PAC-MBR) or GAC post-treatment (MBR-GAC) was observed to significantly complement MBR treatment to obtain high overall removal of less hydrophobic and biologically resistant trace organics. In both systems, the well known problematic compound carbamazepine (neutral charge) consistently registered high removal, while fenoprop and diclofenac (negatively charged) showed high resistance. However, differences in breakthrough profiles of the neutral compound metronidazole and the negatively charged compounds 
ketoprofen and naproxen between MBR - GAC and PAC - MBR systems were noticeable. PAC - MBR offered better performance in terms of activated carbon consumption.

\section{Acknowledgement}

A postgraduate scholarship to Luong Nguyen from the University of Wollongong, Australia and the Thanh Hoa provincial government (Vietnam) is greatly appreciated. Mitsubishi Rayon Engineering, Japan, Activated Carbon Technologies Pty Ltd, Australia and Australian Nuclear Science and Technology Organisation (ANSTO) are thanked for the provision to membrane module, PAC samples, and analysis of PAC properties, respectively.

\section{References}

[1] Abegglen, C., Joss, A., McArdell, C.S., Fink, G., Schlüsener, M.P., Ternes, T.A., Siegrist, H. 2009b. The fate of selected micropollutants in a single-house MBR. Water Res, 43, 20362046.

[2] Bernhard, M., Maller, J., Knepper, T.P. 2006. Biodegradation of persistent polar pollutants in wastewater: Comparison of an optimised lab-scale membrane bioreactor and activated sludge treatment. Water Res, 40, 3419-3428.

[3] Hai, F.I., Tessmer, K., Nguyen, L.N., Kang, J., Price, W.E., Nghiem, L.D. 2011. Removal of micropollutants by membrane bioreactor under temperature variation. J. Membr Sci, 383, $144-151$.

[4] Hernández-Leal, L., Temmink, H., Zeeman, G., Buisman, C.J.N. 2011. Removal of micropollutants from aerobically treated grey water via ozone and activated carbon. Water Res, 45, 2887-2896. 
[5] Kim, S.D., Cho, J., Kim, I.S., Vanderford, B.J., Snyder, S.A. 2007. Occurrence and removal of pharmaceuticals and endocrine disruptors in South Korean surface, drinking, and waste waters. Water Res, 41, 1013-1021.

[6] Li, X., Hai, F.I., Nghiem, L.D. 2011. Simultaneous activated carbon adsorption within a membrane bioreactor for an enhanced micropollutant removal. Bioresour Technol, 102, $5319-5324$.

[7] Lipp, P., Groay, H.-J., Tiehm, A. 2012. Improved elimination of organic micropollutants by a process combination of membrane bioreactor (MBR) and powdered activated carbon (PAC). Desalin. Water Treat, 42, 65-72.

[8] Ng, C. A., Sun, D., Fane, A.G. 2006. Operation of Membrane Bioreactor with Powdered Activated Carbon Addition. Sep Sci Technol, 41, 1447-1466.

[9] Nguyen, L.N., Hai, F.I., Kang, J., Price, W.E., Nghiem, L.D. 2012. Removal of trace organic contaminants by a membrane bioreactor - granular activated carbon (MBR-GAC) system. Bioresour Technol, 113, 169-173.

[10] Radjenovic, J., Petrovic, M., Barceló, D. 2007. Analysis of pharmaceuticals in wastewater and removal using a membrane bioreactor. Anal. Bioanal. Chem, 387, 1365-1377.

[11] Reif, R., Suairez, S., Omil, F., Lema, J.M. 2008. Fate of pharmaceuticals and cosmetic ingredients during the operation of a MBR treating sewage. Desalination, 221, 511-517.

[12] Şeker, Ş., Beyenal, H. and Tanyolaç, A. 1995. The effects of biofilm thickness on biofilm density and substrate consumption rate in a differential fluidizied bed biofilm reactor (DFBBR). J. Biotechnol., 41, 39-47. 
[13] Serrano, D., Suárez, S., Lema, J.M., Omil, F. 2011. Removal of persistent pharmaceutical micropollutants from sewage by addition of PAC in a sequential membrane bioreactor. Water Res, 45, 5323-5333.

[14] Tadkaew, N., Hai, F.I., McDonald, J.A., Khan, S.J., Nghiem, L.D. 2011. Removal of trace organics by MBR treatment: The role of molecular properties. Water Res, 45, 2439-2451.

[15] Ternes, T.A., Meisenheimer, M., McDowell, D., Sacher, F., Brauch, H.-J.r., Haist-Gulde, B., Preuss, G., Wilme, U., Zulei-Seibert, N. 2002. Removal of Pharmaceuticals during Drinking Water Treatment. Environ. Sci. Technol, 36, 3855-3863.

[16] Urase, T., Kagawa, C., Kikuta, T. 2005. Factors affecting removal of pharmaceutical substances and estrogens in membrane separation bioreactors. Desalination, 178, 107113.

[17] Visvanathan, C., Thu, L.N., Jegatheesan, V., Anotai, J. 2005. Biodegradation of pentachlorophenol in a membrane bioreactor. Desalination, 183, 455-464.

[18] William, C. 2009. Carbon Enhanced Membrane Biological Reactors. in: Water Arabia conference. Manama, Kingdon of Bahrain, pp. http://www.sawea.org/pdf/2009/Session/DCarbonEnhancedMBRs.pdf.

[19] Zhang, L., Q., Urase, T., Feng, L. 2008. Removal of carbamazepine in an enhanced membrane bioreactor with small dose addition of powdered activated carbon. in: Advances in Chemical Technologies for Water and Wastewater Treatment, (Eds.) X. Wang, R. Chen, Shaanxi Sci \& Tech Publ House, Shanxi, China, pp. 199-205. 


\section{LIST OF TABLES}

Table 1: TOC and TN removal in MBR, MBR - GAC and PAC - MBR systems.

\begin{tabular}{ccccc}
\hline \multirow{2}{*}{ Parameters } & MBR & MBR - GAC & \multicolumn{2}{c}{ PAC - MBR } \\
\cline { 4 - 5 } TOC & $98 \pm 2(\mathrm{n}=58)$ & $98 \pm 1(\mathrm{n}=28)$ & $98 \pm 1(\mathrm{n}=5)$ & $98 \pm 2(\mathrm{n}=13)$ \\
\hline TN & $46 \pm 15(\mathrm{n}=58)$ & $41 \pm 11(\mathrm{n}=28)$ & $30 \pm 11(\mathrm{n}=5)$ & $53 \pm 13(\mathrm{n}=13)$ \\
\hline
\end{tabular}


Table 2: Removal efficiencies of the trace organic contaminants obtained in this study and corresponding values recorded in the literature.

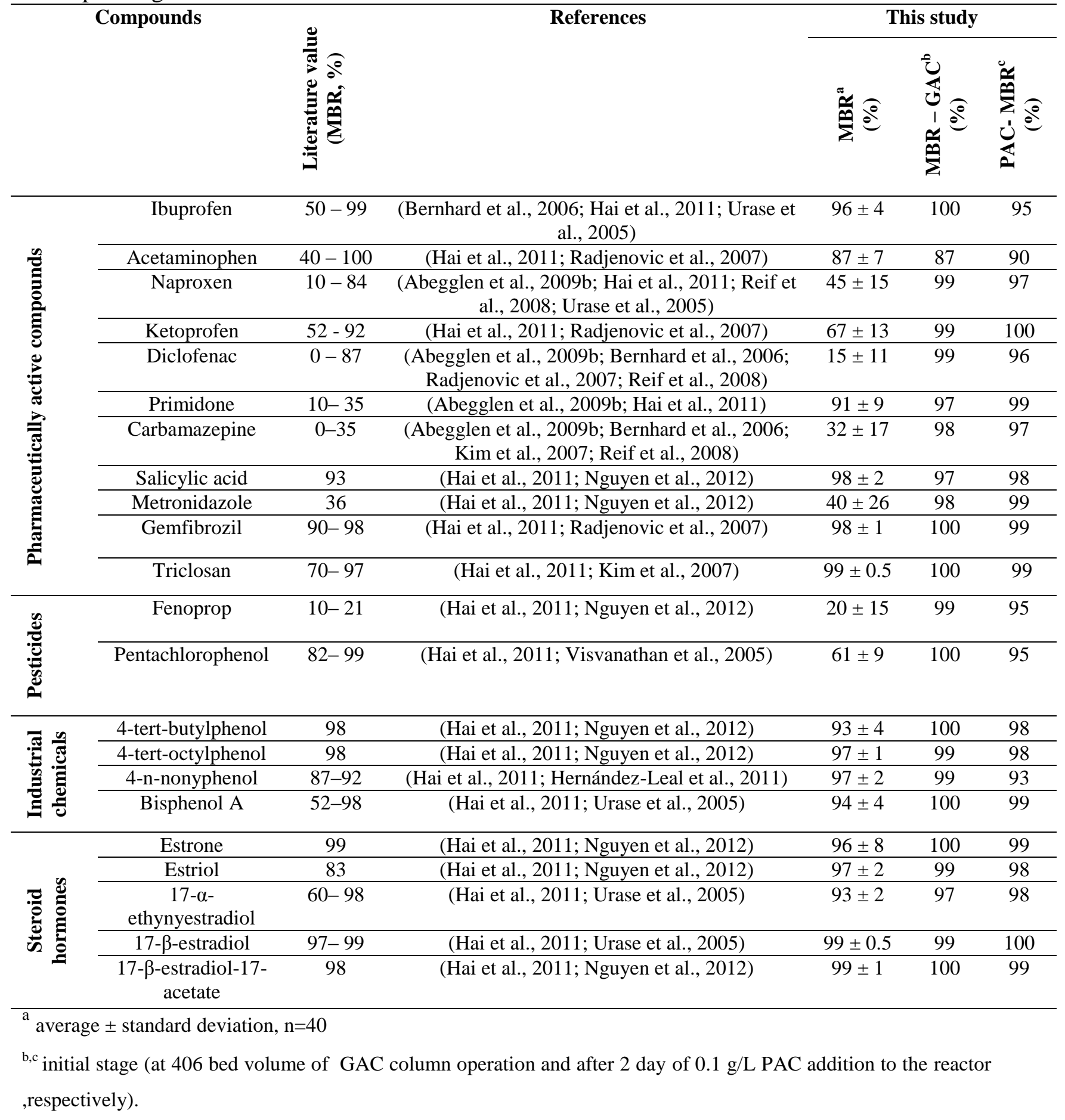




\section{LIST OF FIGURES}

Figure 1: Comparison of removal efficiency of trace organic contaminants by MBR - GAC and PAC - MBR systems at a treated volume of $135 \mathrm{~L}$ over 40, 30 and 30 days of MBR - GAC, PAC - MBR (0.1 g PAC/L) and PAC - MBR (0.5 g PAC/L) operation, respectively. The compounds were arranged in the increasing order of removal by MBR-GAC.

Figure 2: Breakthrough profiles of seven biologically persistent hydrophilic trace organic contaminants as a function of operation time and volume of wastewater treated in the (a) MBR GAC and (b) PAC - MBR (0.5 g PAC/L) systems. The breakthrough values are defined as percentage of the effluent concentration over the influent concentration of the same sampling event.

Figure 3: Comparison of breakthrough profiles of fenoprop as a marker compound in different systems (MBR-GAC, PAC - MBR (0.1 g PAC/L) and PAC - MBR (0.5 g PAC/L)) taking into consideration activated carbon usage rate. 


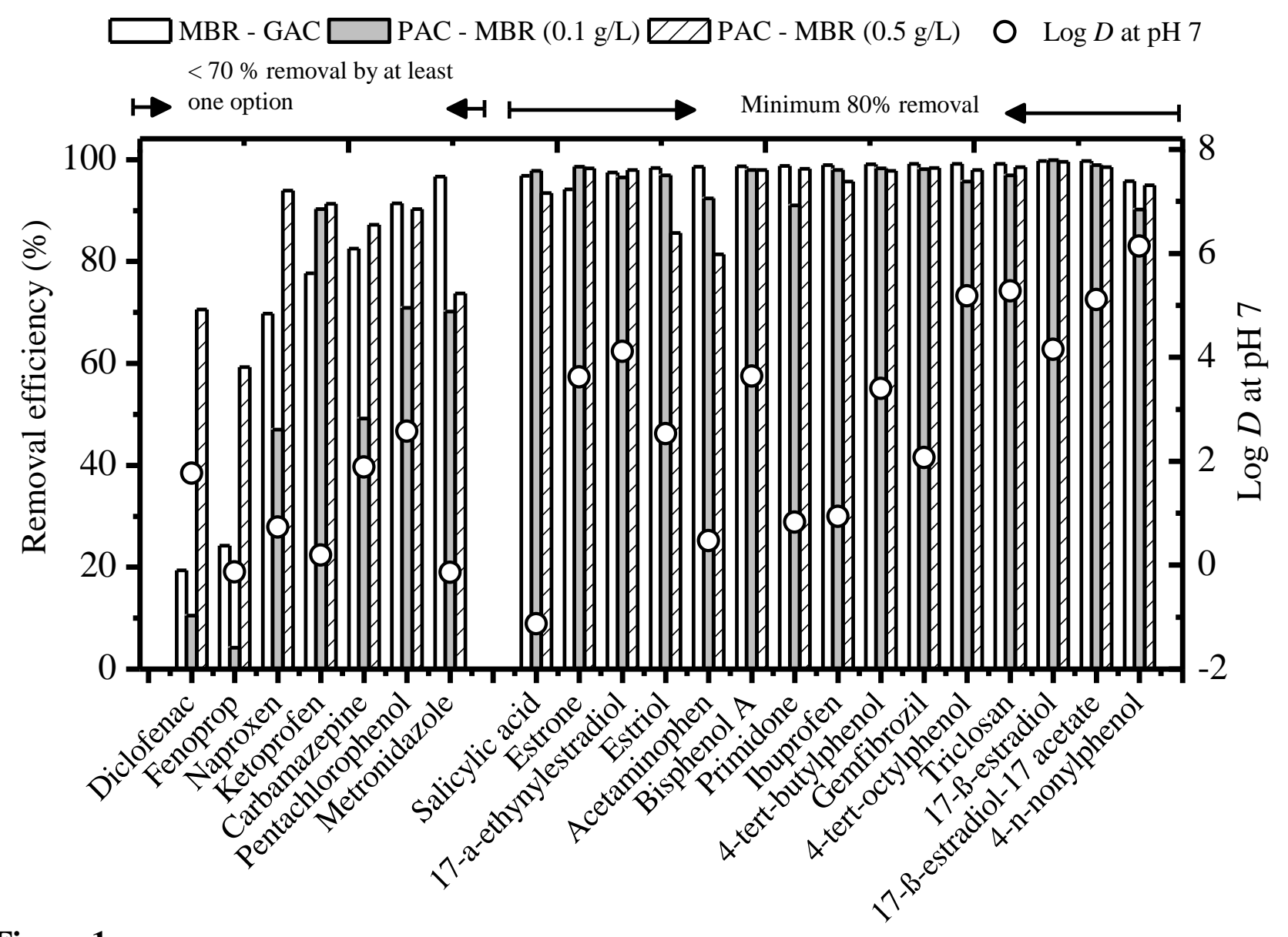

Figure 1 
$\square$ Metronidazole $\oplus$ Fenoprop $\times$ Naproxen
(a) $\downarrow$ Ketoprofen + Diclofenac $\Delta$ Carbamazepine $\triangleright$ Pentachlorophenol
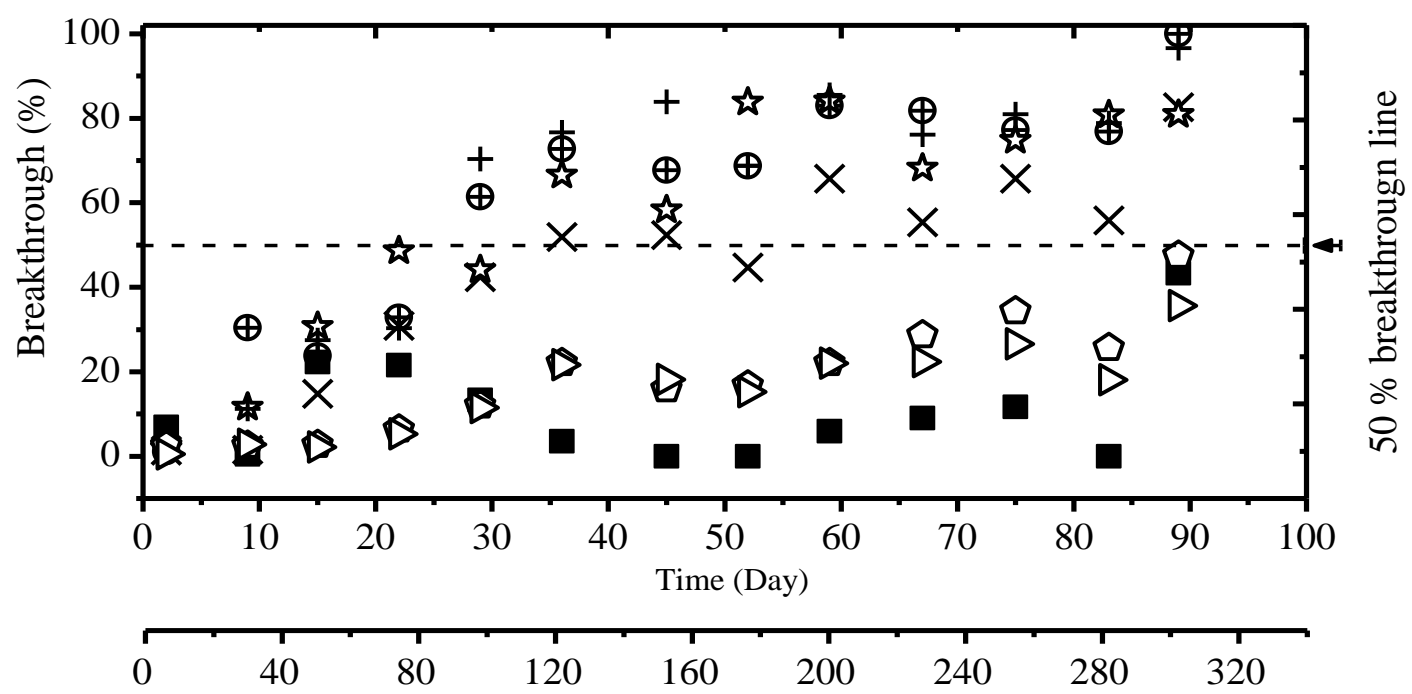

(b)

Treated volume (L)

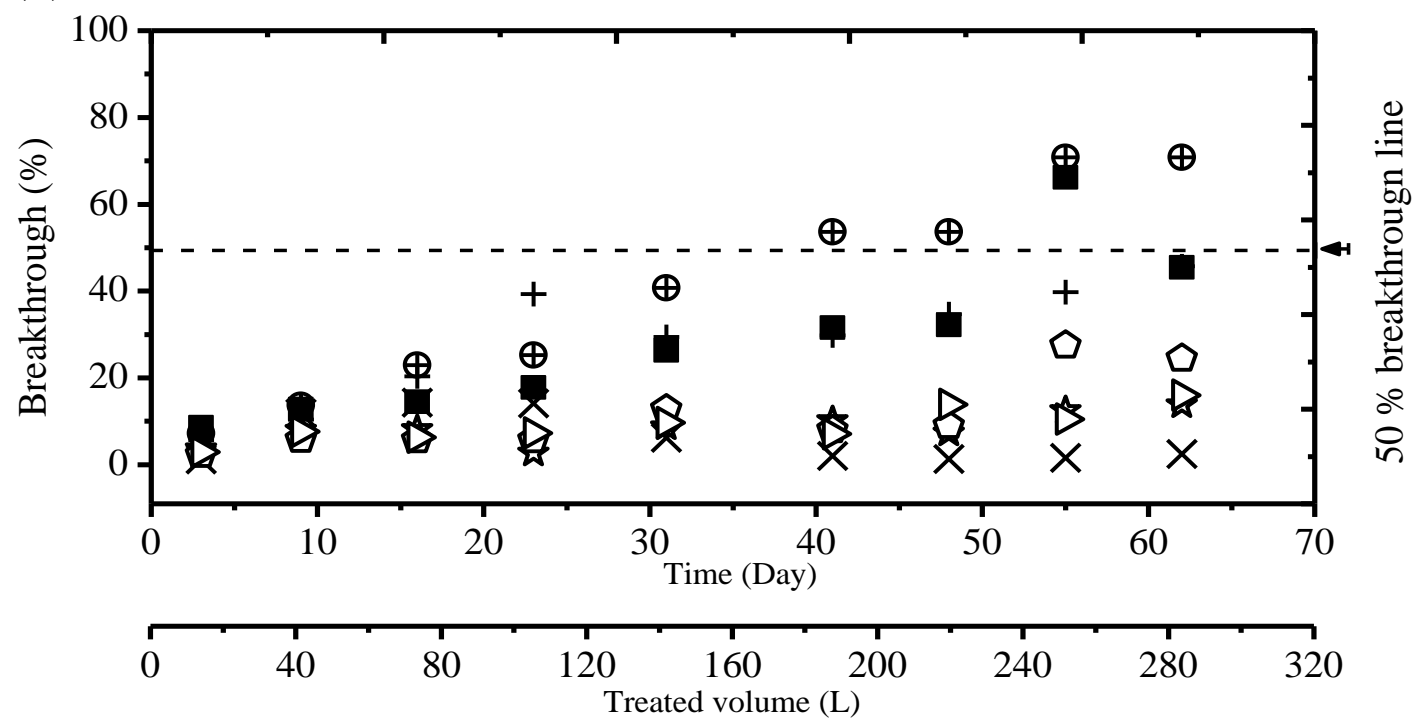

Figure 2 


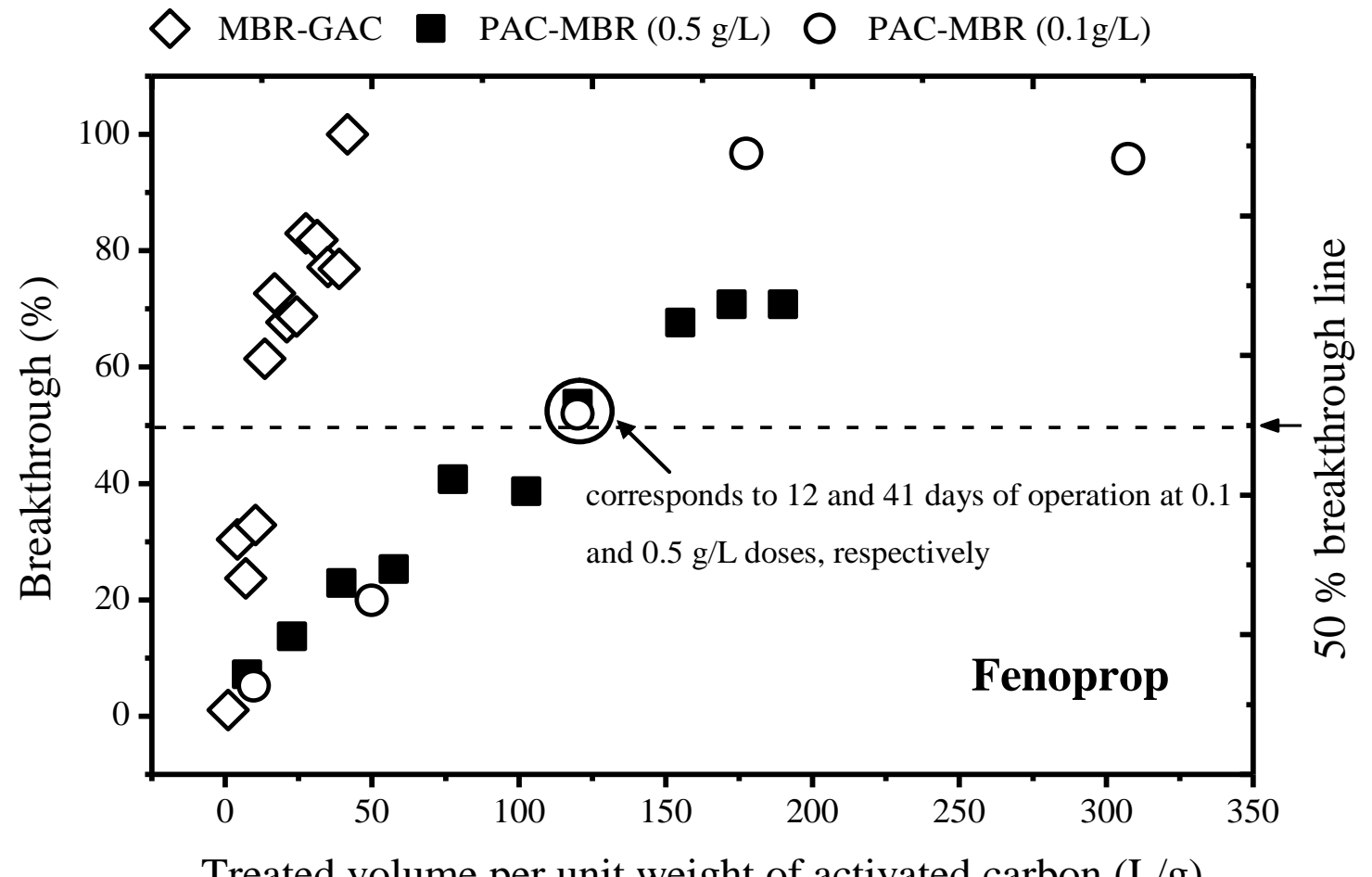

Treated volume per unit weight of activated carbon $(\mathrm{L} / \mathrm{g})$

Figure 3 


\section{Comparison between sequential and simultaneous application of activated carbon with membrane bioreactor for trace organic contaminant removal}

\section{SUPPLEMENTARY DATA}

Luong N. Nguyen ${ }^{\mathrm{a}}$, Faisal I. Hai ${ }^{\mathrm{*}}$, Jinguo Kang
Web
Wenshan $\mathrm{Guo}^{\mathrm{c}}$, Hao H. $\mathrm{Ngo}^{\mathrm{c}}$ and Kuo-Lun Tung ${ }^{\mathrm{d}, \mathrm{e}}$

${ }^{a}$ Strategic Water Infrastructure Laboratory, School of Civil, Mining and Environmental Engineering, University of Wollongong, Wollongong, NSW 2522, Australia

${ }^{\mathrm{b}}$ Strategic Water Infrastructure Laboratory, School of Chemistry, University of Wollongong, Wollongong, NSW 2522, Australia

${ }^{\mathrm{c}}$ Centre for Technology in Water and Wastewater, School of Civil and Environmental Engineering, University of Technology Sydney, Sydney, Broadway, NSW 2007, Australia

${ }^{\mathrm{d}}$ Department of Chemical Engineering, National Taiwan University, Roosevelt Rd, Taipei 106, Taiwan

${ }^{\mathrm{e}} \mathrm{R} \& \mathrm{D}$ Center of Membrane Technology, Chung Yuan University, Chung-Li 320, Taiwan

* Corresponding author: Faisal I. Hai, Email: faisal@uow.edu.au, Ph +61 242213054 
Table S1: Physicochemical properties of the selected trace organics.

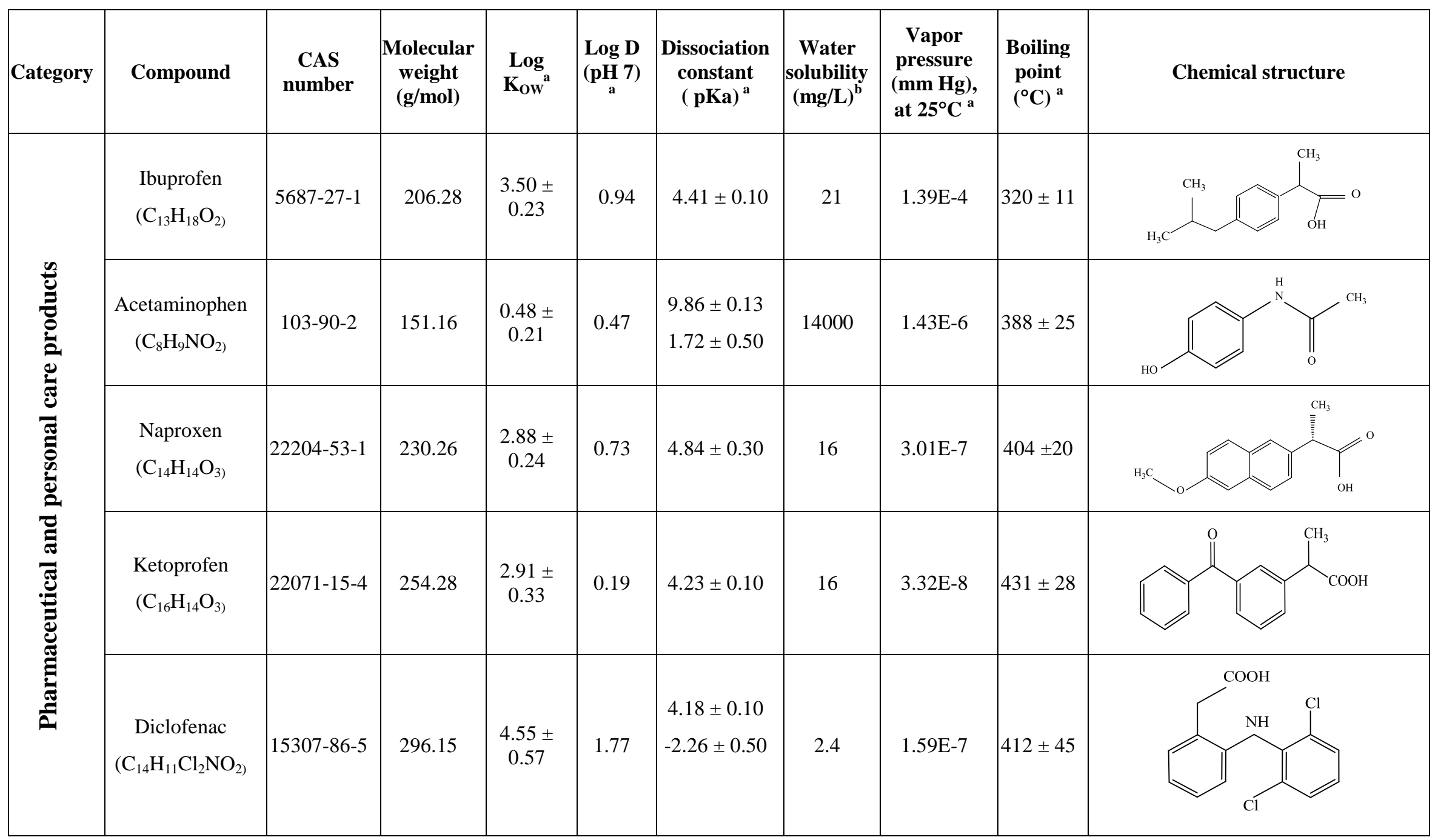




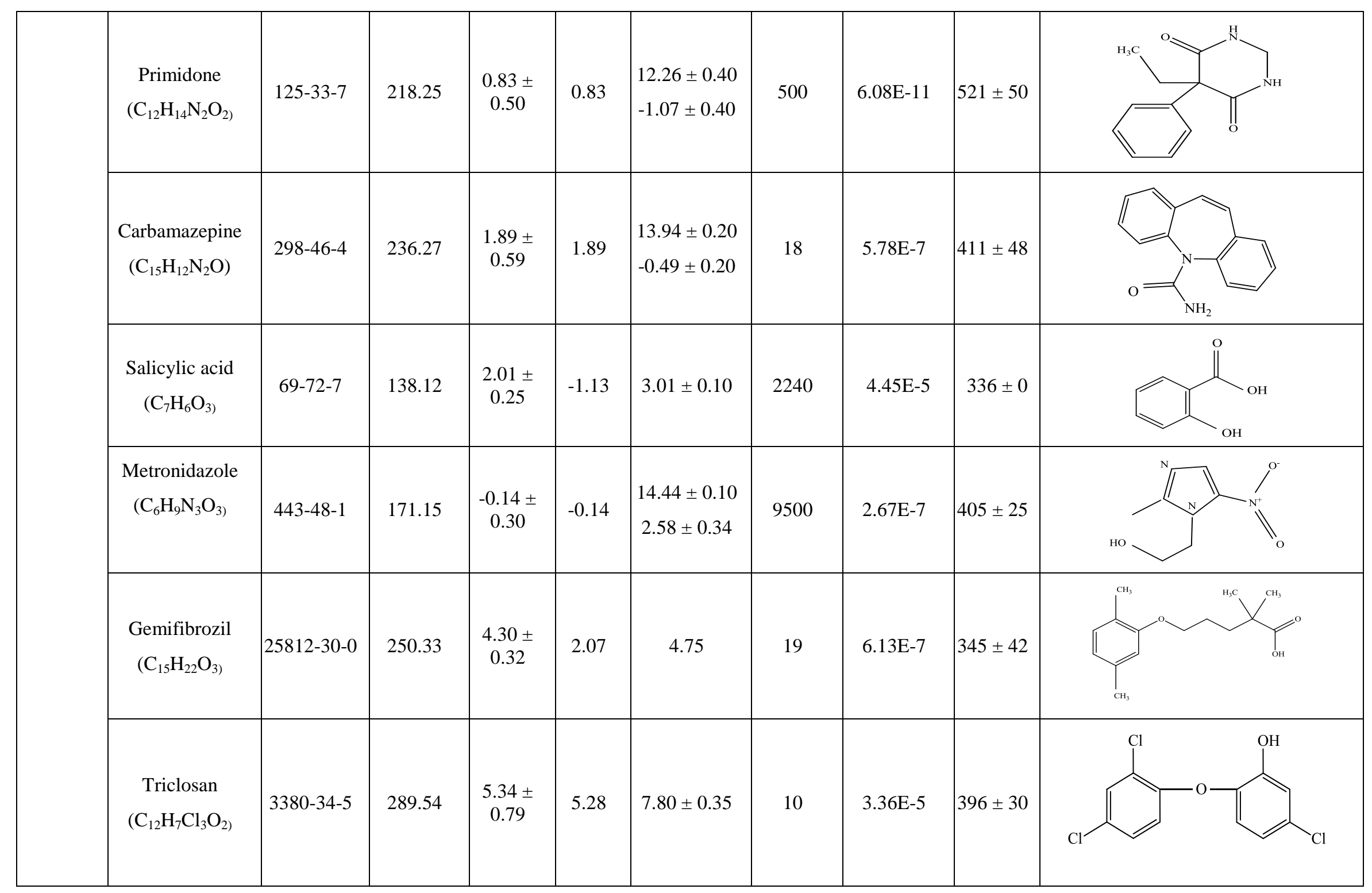




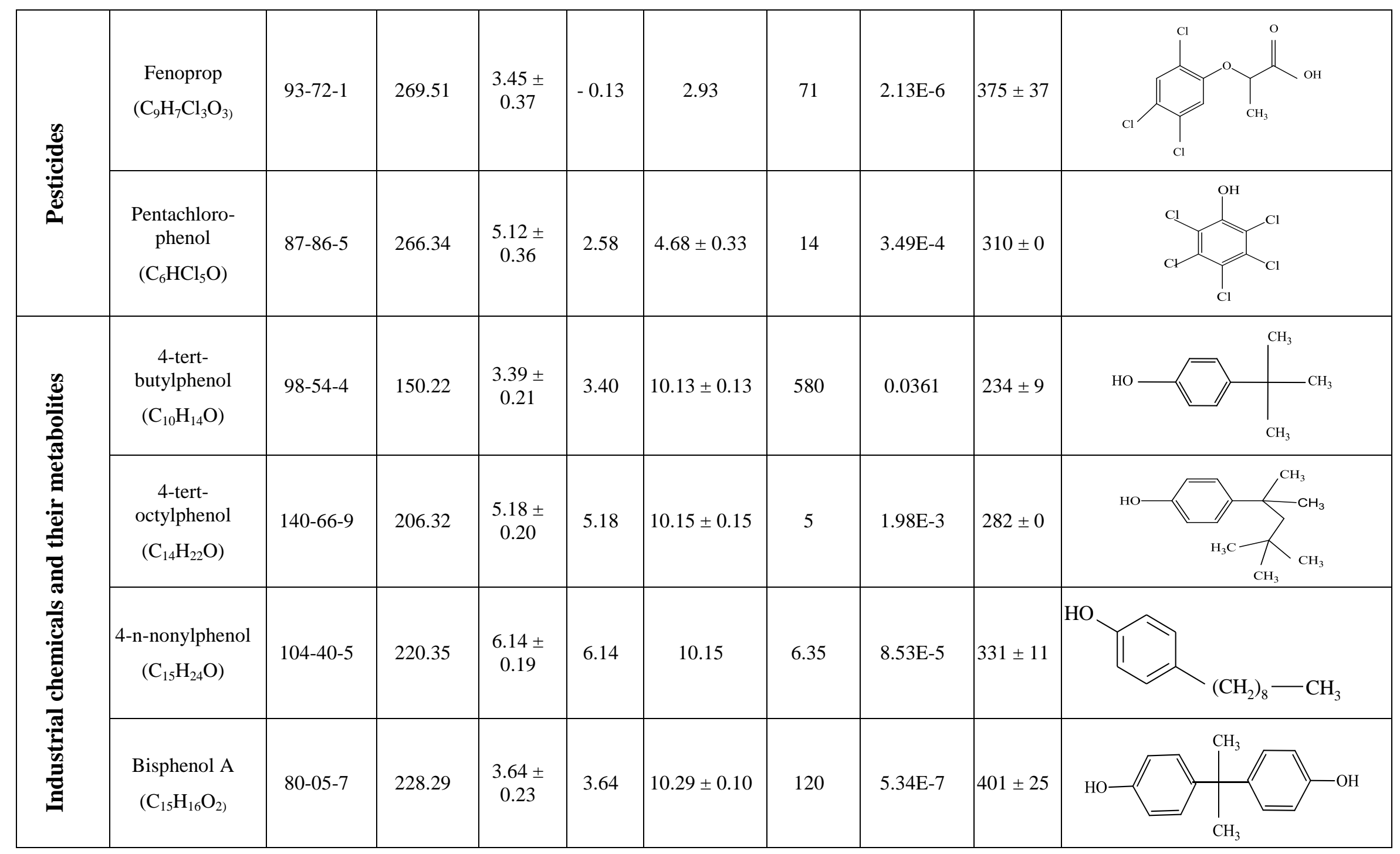




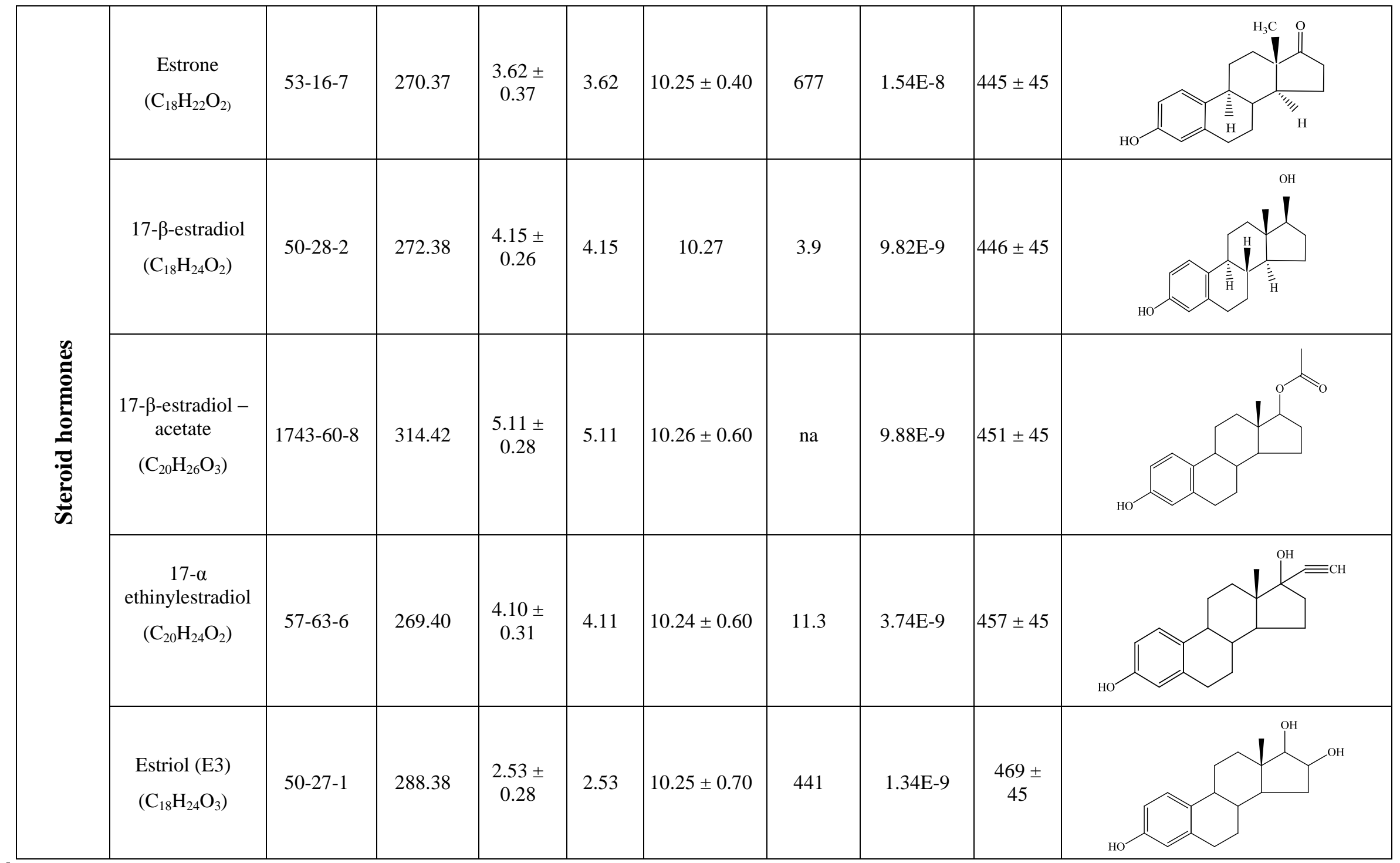

${ }^{a}$ Source: SciFinder database https://scifinder.cas.org/scifinder/view/scifinder/scifinderExplore.jsf

${ }^{\mathrm{b}}$ Source: http://chem.sis.nlm.nih.gov/chemidplus/

na: data not available 
Table S2: Characteristics of PAC-1000 and GAC-1200.

\begin{tabular}{|c|c|c|}
\hline \multirow{2}{*}{ Parameters } & \multicolumn{2}{|c|}{ Values } \\
\hline & PAC & GAC \\
\hline Apparent density $(\mathrm{g} / \mathrm{mL})^{\mathrm{a}}$ & $0.35-0.45$ & $0.42-0.50$ \\
\hline $\begin{array}{c}\text { Specific surface area } \\
(\text { MultiPoint BET m²/g) }\end{array}$ & 1355 & 1121 \\
\hline Ash content $(\%)^{\mathrm{a}}$ & 14 & 3 \\
\hline Iodine number $\left(\mathrm{mg} \text { of } \mathrm{I}_{2} / \mathrm{g}\right)^{\mathrm{a}}$ & $>1000$ & $>1200$ \\
\hline Particle size $^{a}$ & $15-30 \mu \mathrm{m}$ & $\begin{array}{c}6 \times 12 \text { mesh (1.6-2.0 } \\
\mathrm{mm})\end{array}$ \\
\hline Pore volume $(\mathrm{cc} / \mathrm{g})^{\mathrm{b}}$ & 0.228 & 0.043 \\
\hline Pore diameter $(\mathrm{nm})^{b}$ & 3.139 & 3.132 \\
\hline
\end{tabular}

\footnotetext{
${ }^{a}$ Data from Activated Carbon Pty Ltd, Australia.

${ }^{\mathrm{b}}$ Data obtained from a nitrogen adsorption/desorption measurement using an Autosorb iQ. The measurement was conducted at the Australian Nuclear Science and Technology Organisation. Pore volume and pore diameter were calculated based on the Barret-Joyner-Halenda method.
} 

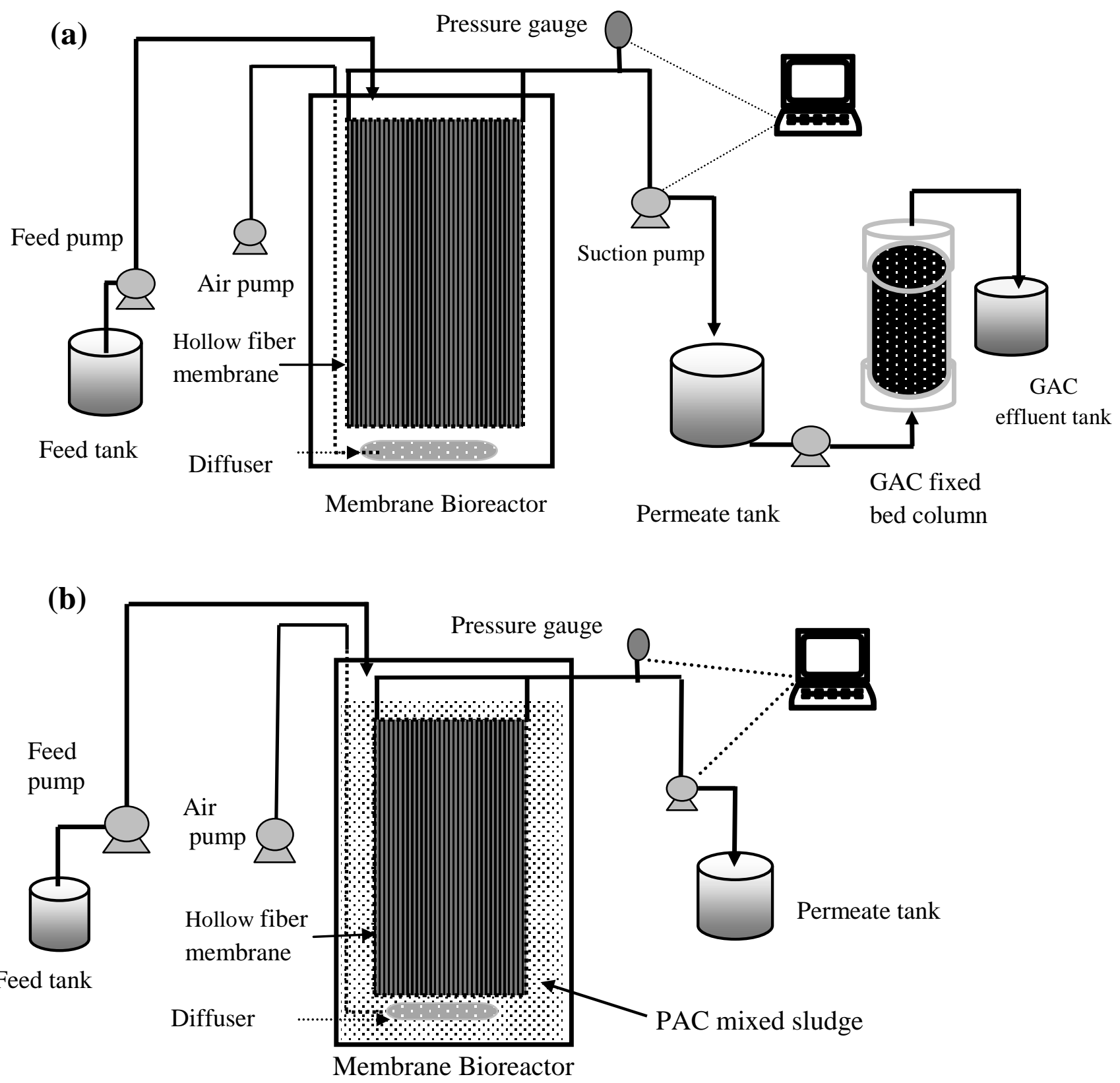

Figure S3: Schematic diagram of (a) MBR-GAC, and (b) PAC - MBR systems.

The MBR system consisted of a glass reactor (active volume of $4.5 \mathrm{~L}$ ), an air pump, a pressure sensor, feed and permeate tanks, influent and effluent pumps and a submerged PVDF hollow fiber membrane module supplied by Mitsubishi Rayon Engineering, Japan. The membrane had a nominal pore size of $0.4 \mu \mathrm{m}$ and a total surface area of $0.074 \mathrm{~m}^{2}$. The membrane module was operated under an average flux of $3.1 \mathrm{~L} / \mathrm{m}^{2} \mathrm{~h}$ on a 14 minute suction and 1 minute rest cycle, resulting in a hydraulic retention time of 24 hours. 
Table S4: Schedule of continuous operation of the MBR systems

\begin{tabular}{cl}
\hline Day & \multicolumn{1}{c}{ Operation mode } \\
\hline $0-51$ & MBR start- up period (without trace organics in feed) \\
\hline $52-65$ & Operation with trace organics in feed \\
\hline $66-158$ & MBR only \\
\hline $159-196$ & MBR - GAC experiment \\
\hline $197-205$ & MBR only \\
\hline $206-242$ & $\begin{array}{l}\text { MBR only (stabilization period after sludge } \\
\text { withdrawal })\end{array}$ \\
\hline $243-306$ & PAC - MBR (0.1 g/L PAC) \\
\hline
\end{tabular}

